\title{
Reflections on the Energy of Black Holes *
}

\author{
Tevian Dray $^{\dagger}$ \\ Department of Mathematics, Oregon State University, Corvallis, OR 97331, USA \\ tevian@math. oregonstate.edu \\ Carlo Rovelli \\ CPT, Aix-Marseille Université, Université de Toulon, CNRS, F-13288 Marseille, FRANCE \\ rovelli@cpt.univ-mrs.fr
}

March 19, 2019

\begin{abstract}
Inside a black hole, there is no local way to say which side of a sphere is the inside, and which is the outside. One can easily be gulled by this fact into mixing up the sign of the energy. We lead the reader astray with a naïve treatment of the energy of a null shell in black hole spacetimes. We then resolve the confusion, showing that global, rather than local, considerations offer good guidance.
\end{abstract}

${ }^{*}$ Essay written for the Gravity Research Foundation 2019 Awards for Essays on Gravitation.

${ }^{\dagger}$ Corresponding author. 


\section{The problem}

What is the total energy associated with a black hole spacetime? Surely, the answer is the parameter $M$ in the line element. For concreteness, let's consider the Schwarzschild geometry. We can model a physical black hole by collapsing a spherically symmetric shell in flat space. For simplicity, we follow Dray and 't Hooft [1], and join Minkowski and Schwarzschild spacetimes along a lightlike, spherically symmetric worldtube, as shown in Figure 1 .

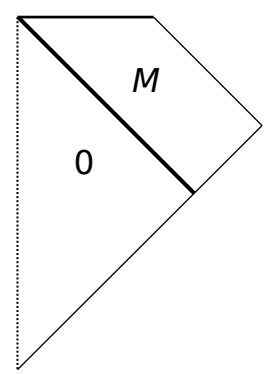

Figure 1: A shell of massless matter in flat space collapsing to form a black hole.

The total energy at any instant of time is given by integrating the energy density over a spacelike hypersurface. The distributional energy density on our shell is clearly $M$, the mass of the resulting black hole.

Dray and 't Hooft [1] also considered the similar situation of a shell collapsing in a black hole background, that is, joining two Schwarzschild spacetimes of different masses. As shown in Figure 2, the result is similar: the energy density of the shell is precisely the difference between the masses of the two Schwarzschild regions. Which way do the signs go? With the regions labeled as in Figure 2, that is, with mass $m$ "before" the shell and mass $M$ "after", the shell has energy density $M-m$.

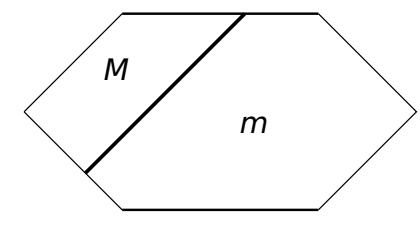

Figure 2: A shell of massless matter joining two black holes.

A somewhat more physical version of the scenario in Figure 2 can be obtained by colliding two shells, replacing the second asymptotic region by the (flat) interior of a collapsing shell. Such a scenario is shown in Figure 3, in which four spacetime regions are joined across two crossing shells. Before the crossing, there is an expanding shell of mass $\mu$ and a collapsing shell of mass $M$. Dray and 't Hooft [1] pointed out that for such shells there is an additional regularity condition at the crossing point, which here becomes

$$
r(r-2(M+\mu))=(r-2 \mu)(r-2 x),
$$




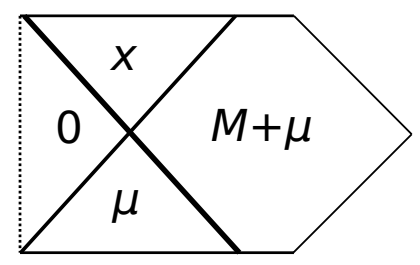

Figure 3: A light, outgoing shell crossing a heavier, ingoing shell.

where $r$ is the Schwarzschild radius of the crossing point and $x$ the mass of the Schwarzschild between the shells after the crossing. For the causal structure shown, $2 \mu<r<2(M+\mu)$. 1]

What is the energy in the two shells after the crossing? It is straightforward to argue that the the upper-left shell carries energy $x$, the mass of the final black hole. Comparing Figure 2 with the corresponding region in Figure 3 - with which it must be locally isomorphic - leads to the conclusion that the energy of the upper-right shell must be $x-(M+\mu)$. Solving for $x$ in Equation (1) leads to

$$
x=\frac{M}{1-\frac{2 \mu}{r}},
$$

so that

$$
x-(M+\mu)=-\mu\left(\frac{1-\frac{2(M+\mu)}{r}}{1-\frac{2 \mu}{r}}\right)>0 .
$$

Thus, all four shells carry positive energy.

Wait a minute! Energy should be conserved. The total energy at late times should match that at early times - the regularity condition at the crossing point ought to ensure that there is no "extra" energy associated with the crossing. But if all four shells carry positive energy, then the total energy at late times is $x+(x-(M+\mu))=2 x-(M+\mu)$ which differs from the energy at early times, which is $M+\mu$. On the other hand, the difference in shell energies at late times is $x-(x-(M+\mu))$, which does equal the energy at early times. Thus, energy conservation implies that the upper-right shell must have negative energy density.

So which is it? Is the shell energy positive or negative? Local considerations appear to require positive energy, to match Figure 2; global considerations appear to require negative energy, to restore energy conservation.

\section{Resolution}

A clue can be found by comparing the collapsing shell model (Figure 1) with the usual Kruskal extension of the Schwarzschild geometry, with two asymptotic regions. For the collapsing shell, Gauss's Law tells us that, on any hypersurface, the total energy inside any sphere equals the gravitational flux through the sphere. This is essentially the mechanism used to compute the ADM mass [2] at spatial infinity: Compute the flux through a large

\footnotetext{
${ }^{1}$ It is also possible to have $r>2(M+\mu)$, but the causal structure is then different.
} 
sphere, then take the limit to infinity. Thus, the total energy on any such hypersurface is $M$, as already claimed. However, for the Kruskal extension, the boundary consists of two spheres, one in each asymptotic region. There is no collapsing shell, and hence no energy, on the hypersurface between them. Thus, the total energy on any hypersurface must be zero.

Furthermore, since the asymptotic region in Figure 1 is isomorphic to one side of the Kruskal extension, the integral over one sphere must be $M$. The integral over the other sphere must therefore be $-M$.

What's going on here? It is tempting to argue that this negative energy on one side is due to the opposite time orientations of the two asymptotic regions, as manifested for instance by examining $t=$ constant surfaces, where $t$ is the Schwarzschild time coordinate. But this argument is clearly insufficient. There is a global time orientation in all of these scenarios, namely up the page. Interchanging the asymptotic regions by horizontal reflection can not change the local physics. Similarly, the local isomorphism between Figure 2 and the corresponding region in Figure 3 is not affected by horizontal reflection.

However, the spatial orientations of the two asymptotic regions are geometric - and do not agree. These opposite spatial orientations clearly suffice to explain the negativity of the computed energy in one region, since an outward flux in one region corresponds to an inward flux in the other. Put differently, the spatial orientation is not invariant under reflection.

Crucially, there is no canonical spatial orientation defined locally inside the black hole. Don't forget, "decreasing radius" has become time! Inside a black hole there is no local sense in which a sphere has a naturally defined inside and outside!

This, then, is the resolution of the apparent paradox: Figure 2 tells us that the energy of the shell is positive as seen in the asymptotic universe in which the shell originated, that is, using the spatial orientation inherited from that region. But that region does not exist in Figure 3; as seen from the only asymptotic region we have, the shell energy is negative. Energy conservation is restored. Similar arguments can be used to show that global energy conservation holds in all of the colliding shell models in [1], including those with two asymptotic regions.

The moral: Always look both ways before crossing!

\section{Acknowledgments}

This essay grew out of a simple question asked by one of us (CR) of the other (TD), which however resulted in a flurry of 50 email messages over the subsequent week, mostly expressing strong disagreement, until finally converging on a resolution

\section{References}

[1] Tevian Dray and Gerard 't Hooft, The Effect of Spherical Shells of Matter on the Schwarzschild Black Hole, Commun. Math. Phys. 99, 613-625 (1985).

[2] Arnowitt R., Deser S., and Misner C. W., Canonical Variables for General Relativity, Phys. Rev. 117, 1595-1602 (1960). 